\title{
Dimeric Boronates Derived from the Reaction of Schiff Bases and Boronic Acids
}

\author{
Victor Barba ${ }^{a}$, Rosa Santillan ${ }^{b}$ and Norberto Farfán ${ }^{*, b}$ \\ ${ }^{a}$ Centro de Investigaciones Químicas, Universidad Autónoma del Estado de Morelos, Av. Universidad 1001, \\ CP 62210 Cuernavaca, Morelos, México \\ ${ }^{b}$ Departamento de Química, Centro de Investigación y de Estudios Avanzados del IPN, 07000, A.P. 14-740, \\ México D. F., México
}

\begin{abstract}
A síntese one-pot de complexos diméricos de boro é descrita. Os compostos foram obtidos pela reação de bases de Schiff (ligantes tridentados) com ácido trans- $\beta$-fenilvinilborônico, ácido 3tiofeninoborônico e ácido metilborônico. A construção das estruturas diméricas é favorecida pela presença de ligações de coordenação $\mathrm{N} \rightarrow \mathrm{B}$ intramoleculares, resultando na formação de anéis heterociclicos de dez membros. A análise cristalográfica de raio $\mathrm{X}$ de um deles confirmou a natureza dimérica desses compostos.
\end{abstract}

The one-pot synthesis of dimeric boron complexes is reported. The compounds were obtained by reaction of Schiff bases (tridentate ligands) with trans- $\beta$-phenylvinylboronic acid, 3thiopheneboronic acid and methylboronic acid. Building of the dimeric structures is favored by the presence of intramolecular $\mathrm{N} \rightarrow \mathrm{B}$ coordination bonds, resulting in the formation of ten-membered ring heterocycles. An X-ray crystallographic analysis for one of them confirmed the dimeric nature of these compounds.

Keywords: tridentate ligands, boron, Schiff base, macrocyclic chemistry

\section{Introduction}

Schiff bases have been used for a long time in the formation of complexes with a variety of transition metals or main group elements, mainly because they can increase the stability of complexes by means of chelate formation. ${ }^{1}$ Tridentate ligands extensively studied contain the ONO donor set atoms, which form strong covalent bonds with the oxygen atoms and coordinative bonds with nitrogen. ${ }^{2}$ Our studies on the reactivity of this class of ligands towards boronic acids have shown that formation of the different oligomeric compounds depends on the structure of the ligand.

For instance, the reaction of arylboronic acids with the Schiff base derived from salicylaldehyde and 2-aminophenol ( $\left.\mathrm{H}_{2} \mathrm{SAP}\right)$, leads to monomeric compounds, ${ }^{3}$ while the ligands derived from ethanolamine $\left(\mathrm{H}_{2} \mathrm{SAE}\right)$, give dimeric compounds in good yields (Scheme 1). ${ }^{4}$ It has been noticed that depending on the substituent at the ethanolamine, monomeric or dimeric compounds can be

* e-mail: jfarfan@ cinvestav.mx obtained; ${ }^{5}$ in contrast, substituents at the aromatic moiety of the boronic acid do not alter the course of the reaction. ${ }^{6}$ Ferrocenyl groups attached to the boronic acid lead to stable dimeric compounds, ${ }^{7}$ however, when the boron atom is joined to $-\mathrm{OH},-\mathrm{NMe}_{2}$ or $-\mathrm{C}_{6} \mathrm{~F}_{5}$ groups, the dimeric compounds decompose in the presence of water to give an oxo-dimeric compound whereby an oxygen atom connects the two boron atoms. ${ }^{8}$

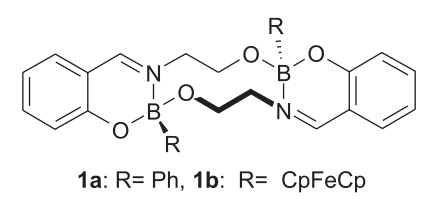

Scheme 1. Dimeric boron complexes derived from $\mathrm{H}_{2} \mathrm{SAE}$ and phenylboronic acid (1a) or ferrocenylboronic acid (1b).

As an extension of our studies, we synthesized nine new boron complexes derived from Schiff base ligands and boronic acids. The ligands used were derived from ethanolamine and salicylaldehyde (2a), 2-hydroxyacetophenone (2b) and 2-hydroxybenzophenone (2c). Ligands $\left(\mathrm{R}^{\prime} \mathrm{LH}_{2}\right)$ were reacted with trans- $\beta$-phenylvinylboronic acid (3a-3c), methylboronic acid (3d-3f), and 
3-thiophenylboronic acid (3g-3i), to give the corresponding dimeric boron complexes (R'LBR").

\section{Results and Discussion}

\section{Synthesis of boron complexes (3a-3i)}

The reaction of ligands $\mathbf{2 a - 2 c}$ with boronic acids was carried out under reflux of methanol. After $1 \mathrm{~h}$ under stirring, boron complexes $\mathbf{3 a - 3 i}$ were obtained in moderate to good yields (55-93\%) (Scheme 2). The dimeric nature of these compounds was established first by mass spectrometry. In all nine cases, the EI-Mass Spectra showed the $[\mathrm{M}-\mathrm{R}]^{+}$ion which corresponds to the loss of the group attached to the boron atom; this pattern is well established in the literature and is characteristic for this type of compounds. ${ }^{5}$ In contrast to other dimeric boron complexes, ${ }^{8}$ compounds $\mathbf{3 a - 3 i}$ are stable to hydrolysis, this is attributed to the presence of covalent and coordinative bonds around the boron atom, as well as the non labile nature of the substituent attached to the boron atom. The new compounds are slightly more soluble in organic solvents than similar systems, ${ }^{4}$ the most soluble ones are the acetophenone derivatives. It should be mentioned that acetophenone derivatives have the lower melting points for the series (below $200{ }^{\circ} \mathrm{C}$ ) while those of the other compounds are in the range from $240{ }^{\circ} \mathrm{C}$ to $300{ }^{\circ} \mathrm{C}$.

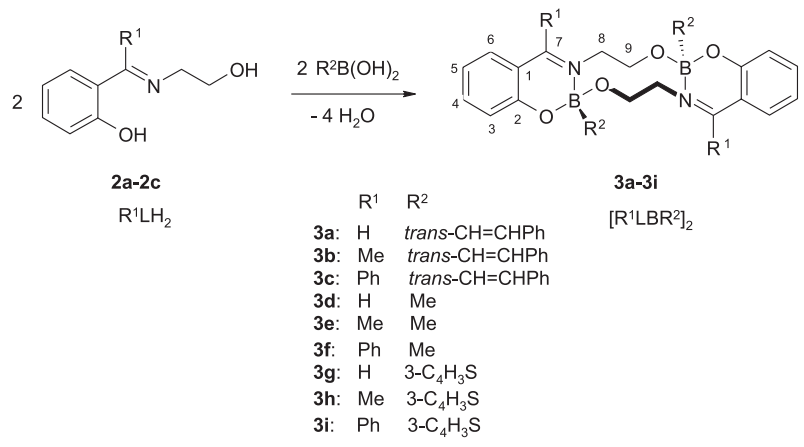

Scheme 2. Synthesis of dimeric boron complexes $\left[\mathrm{R}^{\prime} L B R \text { " }\right]_{2}$.

\section{Spectroscopic trends}

IR spectra show bands between 1606 and $1640 \mathrm{~cm}^{-1}$ assigned to the stretching band of the $\mathrm{C}=\mathrm{N}$ group. The higher values $\left(1638-1640 \mathrm{~cm}^{-1}\right)$ correspond to complexes of the salicylaldehyde derivatives, while those of 2-hydroxybenzophenone (1606-1612 $\left.\mathrm{cm}^{-1}\right)$ appear at lower values. The lower shift observed in $\mathbf{3 c}, \mathbf{3 f}$ and $\mathbf{3 i}$, is attributed to electronic effects of the phenyl groups present in the azomethine group. ${ }^{7}$ In all nine cases the NMR spectra showed signals for only half of the molecule, owing to the symmetry of the compounds in solution. For instance, the ${ }^{1} \mathrm{H}$ NMR spectra show simple signals at $\delta 8.40,8.46$ and $8.29 \mathrm{ppm}$ corresponding to the azomethine group of salicylaldehyde derivatives $\mathbf{3 a}, \mathbf{3 d}$ and $\mathbf{3 g}$, all of them shifted to lower fields in comparison to the ligand $(\delta 8.1)$, due to nitrogen - boron coordination. 2D NMR spectra were recorded when necessary to establish the correct assignment of the compounds. In contrast to the two triplet signals observed for the methylene groups in the ligands; complexes 3a-3i, showed diastereotopic signals for the $\mathrm{CH}_{2}$ groups which are observed as an $\mathrm{AA}^{\prime} \mathrm{XX}$ ' system due to ring closure. NOESY spectra allowed the correct assignment and it was observed that the $\Delta \delta$ is larger for the hydrogens assigned as $\mathrm{H} 9$ in comparison with $\mathrm{H} 8$. The ${ }^{13} \mathrm{C}$ NMR spectra are similar when complexes derived from the same ligand are compared; however, there are significant differences in the chemical shift of the azomethine group when salicylaldehyde, acetophenone and benzophenone complexes are compared. For salicylaldehyde derivatives, this group is shifted to lower frequency (164.6-168.4 ppm) compared to acetophenone and benzophenone derivatives (170.7 to $181.6 \mathrm{ppm}$ ) due to the inductive effect of the methyl and phenyl groups. Furthermore, the signal assigned to C9 in salicylaldehyde derivatives, is shifted to low frequency ( $\Delta \delta \sim 1 \mathrm{ppm})$, in comparison with the signal for C8. In contrast, for benzophenone and acetophenone derivatives, the signal for $\mathrm{C} 8$ is shifted to low frequency $(\Delta \delta \sim 10 \mathrm{ppm})$ in comparison with the signal for C9 due a $\gamma$-gauche effect. ${ }^{9}$ As can be seen in Scheme 2, ligands are joined to the boron atoms through three covalent bonds which include two oxygens and one carbon atom, additionally the nitrogen atom acts as a donor to form a coordination bond the boron atom, which is the Lewis acid. As a result, boron atoms are in a tetrahedral environment. The tetrahedral character of the boron atoms was evidenced from the chemical shifts observed in the ${ }^{11} \mathrm{~B}$ NMR spectra, in all cases the signals appear from 2.7 to $11.5 \mathrm{ppm}$, which is characteristic for this type of complexes. ${ }^{4-8}$ The presence of the two $\mathrm{N} \rightarrow \mathrm{B}$ coordination bonds leads to formation of two six-membered and one ten-membered ring in the complexes.

\section{X-ray crystallographic analysis}

Crystals suitable for X-ray structure analysis of compound $\mathbf{3 g}$ were grown by slow evaporation of a concentrated $\mathrm{C}_{6} \mathrm{H}_{6}$ solution from the starting materials. ${ }^{10}$ Figure 1a shows the molecular structure for $\mathbf{3 g}$ and confirms the dimeric nature of this compound. The crystal structure contains two independent molecules and one molecule of 
benzene in the unit cell. As observed for analogous systems, ${ }^{5}$ the molecule has a crystallographic inversion center and belongs to the $\mathrm{Ci}$ point group, which is in accordance with the symmetry observed in solution by NMR. Furthermore, the two 3-thiophenyl moieties are oriented in a trans disposition to each other, as a result of the symmetry. As shown in Figure 1b, the ten-membered heterocycle has a chair-boat-chair conformation, while the six-membered ring is almost planar. The planarity of the six-membered ring can be seen from the torsion angle values for the C6-C7-N1-B1 $\left(6.37^{\circ}\right)$ and C6-C1-O1-B1 $\left(15.94^{\circ}\right)$ fragments, which are larger than those of compound 1a (3.75 and $12.69^{\circ}$, respectively).
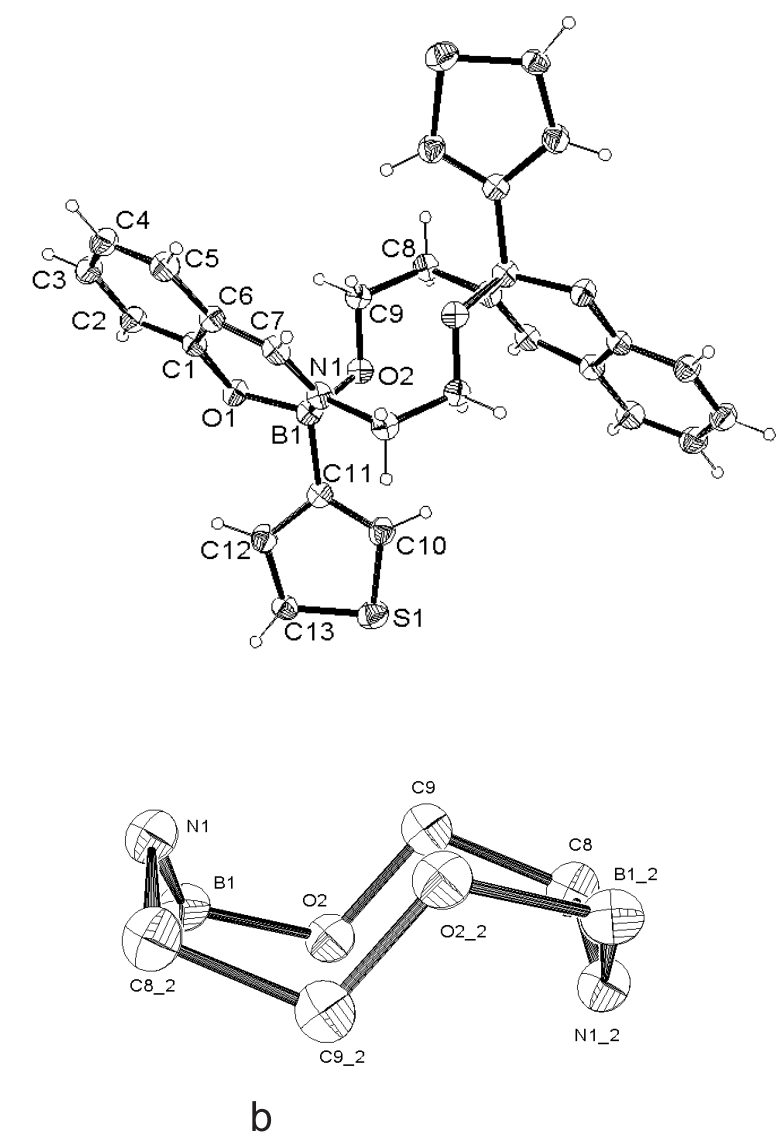

Figure 1. (a) Molecular structure of compound 3g; (b) View of the conformation for the ten-membered ring.

A comparison of bond distances and angles between $\mathbf{3 g}$ and compounds $\mathbf{1 a}$ and $\mathbf{1 b}$ shows no significant differences, therefore, the electronic or steric effects of the substituens attached to the boron atom should be relatively small. Selected bond distances and angles for $\mathbf{3 g}$ are depicted in Table 1. For example, the coordination bond between boron and the imino nitrogen atom has a bond distance of 1.625(4)/1.628(4) $\AA$, which is comparable with that observed for compounds 1a and $\mathbf{1 b}$ (1.624(3) and 1.632
(5) A, respectively). As described above, the boron atoms are in a tetrahedral environment, nonetheless it is distorted as deduced from the angles around the boron atom which are in the range from 106.9 to $112.4^{\circ}$. There are also some differences in the angle values, for instance, the N1-B1C11 angle for compound $\mathbf{3 g}$ has a value of 112.4(2)/ $112.5(2)^{\circ}$ which is larger in comparison with that observed for $\mathbf{1 a}$ and $\mathbf{1 b}$ (108.1(2) and 107,0(3) ${ }^{\mathbf{o}}$, respectively). In contrast, the O2-B1-C11 angle decreases in 3g (109.5(2)/ $\left.109.7(2)^{\circ}\right)$ in relation to the angles observed for $\mathbf{1 a}$ and $\mathbf{1 b}$ (111.9(2) and 110.6(4) $)^{\circ}$, respectively).

Table 1. Selected bond distances $(\AA)$ and angles $\left(^{\circ}\right)$ for compound $3 \mathrm{~g}^{\mathrm{a}}$

\begin{tabular}{lll}
\hline Bond distances $(\AA)$ & Molecule 1 & Molecule 2 \\
$\mathrm{B}(1)-\mathrm{O}(1)$ & $1.480(4)$ & $1.477(4)$ \\
$\mathrm{B}(1)-\mathrm{O}(2)$ & $1.442(6)$ & $1.441(4)$ \\
$\mathrm{B}(1)-\mathrm{N}(1)$ & $1.625(4)$ & $1.628(4)$ \\
$\mathrm{B}(1)-\mathrm{C}(11)$ & $1.610(5)$ & $1.615(5)$ \\
$\mathrm{O}(1)-\mathrm{C}(1)$ & $1.340(4)$ & $1.342(4)$ \\
$\mathrm{O}(2)-\mathrm{C}(9)$ & $1.412(4)$ & $1.401(4)$ \\
$\mathrm{N}(1)-\mathrm{C}(7)$ & $1.287(4)$ & $1.284(4)$ \\
Angles $\left({ }^{\circ}\right)$ & & \\
$\mathrm{O}(2)-\mathrm{B}(1)-\mathrm{O}(1)$ & $112.1(3)$ & $112.3(3)$ \\
$\mathrm{O}(2)-\mathrm{B}(1)-\mathrm{N}(1)$ & $108.3(2)$ & $108.3(2)$ \\
$\mathrm{O}(1)-\mathrm{B}(1)-\mathrm{N}(1)$ & $106.9(2)$ & $107.0(2)$ \\
$\mathrm{O}(2)-\mathrm{B}(1)-\mathrm{C}(11)$ & $109.5(2)$ & $109.7(2)$ \\
$\mathrm{O}(1)-\mathrm{B}(1)-\mathrm{C}(11)$ & $107.6(3)$ & $107.4(2)$ \\
$\mathrm{N}(1)-\mathrm{B}(1)-\mathrm{C}(11)$ & $112.4(2)$ & $112.5(2)$ \\
$\mathrm{C}(1)-\mathrm{O}(1)-\mathrm{B}(1)$ & $124.6(2)$ & $124.6(3)$ \\
$\mathrm{C}(9)-\mathrm{O}(2)-\mathrm{B}(1)$ & $118.6(2)$ & $118.9(3)$ \\
\hline
\end{tabular}

${ }^{a}$ There are two independent molecules in the asymmetric unit.

Two types of hydrogen interactions are presents in the molecule. There are, two intramolecular $\mathrm{C}-\mathrm{H} \cdots \mathrm{O}$ interactions that connect $\mathrm{CH}_{2}$ group with an oxygen atom of the opposite side, with a distance of $2.54 \AA$ (Figure 2a). This interaction is weaker than that in compound $\mathbf{1 a}$, whereby the distance is of $2.43 \AA .{ }^{11}$ The above trans annular interactions are probably present in solution, and may be responsible for the shift to high frequency observed for one of the hydrogens at position $\mathrm{C} 9$, in the ${ }^{1} \mathrm{H}$ NMR spectra. It can be seen in figure $1 \mathrm{~b}$ that this interaction is favored by the close disposition of $\mathrm{O} 2$ and $\mathrm{C} 9$. Moreover, at the intermolecular level, $\mathrm{CH} \cdots \mathrm{O}$ interactions were also found (Figure 2b) in this case between the hydrogen of the azomethine group and the oxygen atom of the tenmembered heterocycle (distance $2.52 \AA$ ).

In summary, stable boron compounds were obtained in a one step synthesis. As mentioned above, the reaction pathway for the formation of the dimeric products is neither affect by the presence of different substituents at the boron atom, nor by substituents at the azomethine group. In 


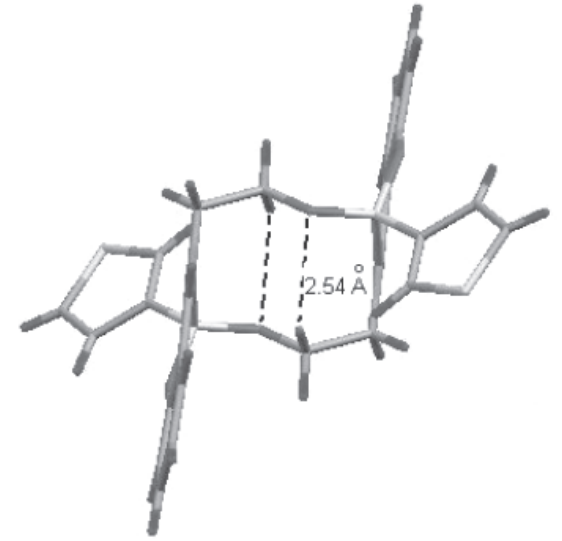

a

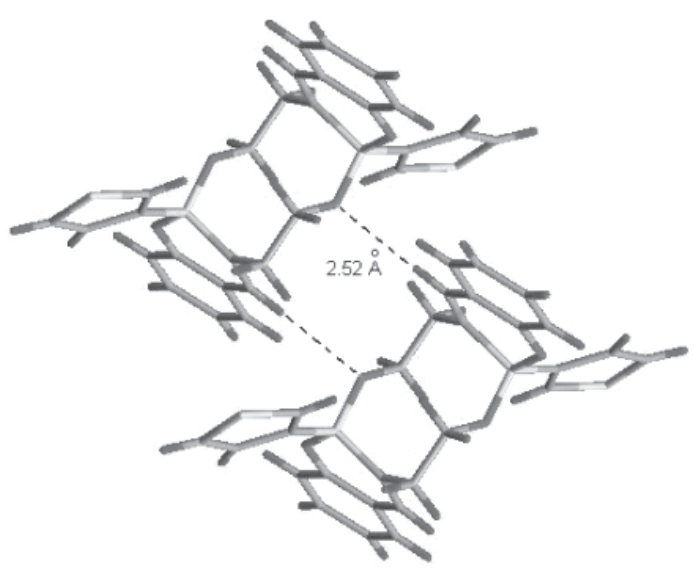

b

Figure 2. (a) Intramolecular hydrogen interactions; (b) Intermolecular hydrogen interactions.

addition, the stability of these compounds is increased, compared to analogous systems. ${ }^{8}$

\section{Instrumental}

NMR spectra were recorded at room temperature using a Bruker 300 spectrometer. Chemical shifts are given in ppm. Infrared spectra have been recorded on a Perkin Elmer 16F-PC FT-IR spectrophotometer. Electronic Ionization mass spectra were obtained with a HP 5989-A mass spectrometer operating in the electron ionization mode. Melting points were determined with a Gallenkamp MFB595 apparatus.

\section{$X$-ray crystallography}

Crystal structure determination of $\mathbf{3 g}$. Intensity data were collected at $293 \mathrm{~K}$ with an Enraf-Nonius CAD4 diffractometer, Mo $\mathrm{K} \alpha$-radiation, $\lambda=0.71073 \AA$, graphite monochromator. Empirical absorption corrections
(DIFABS) were applied. The structure was solved by direct methods (SHELXS-86) ${ }^{12}$ and refined using SHELXL-97. ${ }^{13}$ All non-hydrogen atoms were refined anisotropically. Hydrogen atoms were placed in geometrically calculated positions using a riding model. Crystallographic data have been deposited at the Cambridge Crystallographic Data Center as supplementary material No. 254667. Copies of the data can be obtained free of charge on application to CCDC, 12 Union Road, Cambridge CB2 1EZ, UK. E-mail: deposit@ccdc.cam.ac.uk.

Preparative part

All reagents and solvents were purchased from Aldrich and used without further purification. Ligands $\mathbf{2 a}$ and $\mathbf{2 b}$ were synthesized in accordance to the literature. ${ }^{14}$

Synthesis of ligand $\mathbf{2 c}$

2c was prepared from $1.50 \mathrm{~g}(25.2 \mathrm{mmol})$ of ethanolamine and $5.00 \mathrm{~g}(25.2 \mathrm{mmol})$ of 2-hydroxybenzophenone in $20 \mathrm{ml}$ of ethanol. After 30 minutes under reflux, the solvent was removed under high vacuum. A yellow solid was obtained and washed with hexane (4.7 g, 90\%); mp 85-87 ${ }^{\circ} \mathrm{C}$; IR $v_{\max } / \mathrm{cm}^{-1} 2636,2624,2614$, 2502, 1606(C=N), 1598, 1584, 1570, 1554, 1488, 1474, 1466, 1064, 1022, 774, 700 (KBr); ${ }^{1} \mathrm{H}$ NMR $(300 \mathrm{MHz}$, $\left.\mathrm{CDCl}_{3}\right) \delta$ 7.52-746 (m, 3H H-o, $p$ ), $7.25\left(\mathrm{td},{ }^{3} \mathrm{~J} 7.6\right.$ and ${ }^{4} \mathrm{~J}$ $1.5 \mathrm{~Hz}, 1 \mathrm{H}, \mathrm{H}-4), 7.23-7.19$ (m, 2H, H-m), $6.93\left(\mathrm{~d},{ }^{3} J 7.6 \mathrm{~Hz}\right.$, $1 \mathrm{H}, \mathrm{H}-3$ ), 6.78 (dd, ${ }^{3} J 7.6$ and $\left.{ }^{4} J 1.5 \mathrm{~Hz}, 1 \mathrm{H}, \mathrm{H}-6\right), 6.59\left(\mathrm{t},{ }^{3} J\right.$ $7.6 \mathrm{~Hz}, 1 \mathrm{H}, \mathrm{H}-5), 3.84\left(\mathrm{t},{ }^{3} J 5.3 \mathrm{~Hz}, 2 \mathrm{H}, \mathrm{H}-9\right), 3.46\left(\mathrm{t},{ }^{3} J 5.3\right.$ $\mathrm{Hz}, 2 \mathrm{H}, \mathrm{H}-8$ ) ppm. ${ }^{13} \mathrm{C} \mathrm{NMR}\left(75 \mathrm{MHz}, \mathrm{CDCl}_{3}\right.$ ) $\delta 176.0$ (C7), 164.0 (C-2), 133.4 (C-i), 133.1 (C-4), 131.7 (C-6), 129.3 (C-p), 128.8 (C-o), 127.5 (C-m), 119.3 (C-1), 118.7 (C-3), 117.0 (C-5), 62.1 (C-9), 53.3 (C-8). $m / z(\%): 241\left(\mathrm{M}^{+}, 57\right)$, 240 (44), 224 (43), 210 (100), 196 (33), 152 (26), 107 (47), $91(89)$.

General method for the preparation of boron complexes (3a-3i)

Compounds 3a-3i were synthesized from two equivalents of the corresponding ligand (2a-2c), and two equivalents of the boronic acid derivative using $\mathrm{MeOH}$ as solvent. After 1 hour under refluxed, the water formed during the reaction and part of the solvent were removed with a Dean-Stark tramp. Finally, the solvent was completely removed using a vacuum pump and the product was washed with several portions of hexane. Yellow powders were obtained in moderate yields (55 to $93 \%$ ).

$\left[\mathrm{HLBCH}=\mathrm{CHPh}_{2}(3 \mathrm{a}) .(0.23 \mathrm{~g}, 55 \%) ; \mathrm{mp} 254-256^{\circ} \mathrm{C}\right.$; 
Found: C, 73.37; H, 5.47; N, 4.89\%. Calc. For $\mathrm{C}_{34} \mathrm{H}_{32} \mathrm{~B}_{2} \mathrm{~N}_{2} \mathrm{O}_{4}: \mathrm{C}, 73.67 ; \mathrm{H}, 5.78 ; \mathrm{N}, 5.05 \%$; IR $v_{\max } / \mathrm{cm}^{-1}$ : 2918, 2850, $1640(\mathrm{C}=\mathrm{N}), 1560,1482,1312,1236,1140$, $1114(\mathrm{KBr}) ;{ }^{1} \mathrm{H}$ NMR $\left(300 \mathrm{MHz}, \mathrm{CDCl}_{3}\right) \delta 8.40(\mathrm{~s}, 1 \mathrm{H}, \mathrm{H}-$ 7), 7.55 (ddd, ${ }^{3} J 8.5,{ }^{3} J 7.3$ and $\left.{ }^{4} J 1.6 \mathrm{~Hz}, 1 \mathrm{H}, \mathrm{H}-4\right), 7.45$ (dd, ${ }^{3} J 7.3$ and $\left.{ }^{3} J 1.6 \mathrm{~Hz}, 1 \mathrm{H}, \mathrm{H}-6\right), 7.41\left(\mathrm{~d},{ }^{3} J 7.2 \mathrm{~Hz}, 2 \mathrm{H}, \mathrm{H}-\right.$ o), $7.30\left(\mathrm{t},{ }^{3} \mathrm{~J} 7.2 \mathrm{~Hz}, 2 \mathrm{H}, \mathrm{H}-m\right), 7.19\left(\mathrm{t},{ }^{3} J 7.2 \mathrm{~Hz}, 1 \mathrm{H}, \mathrm{H}-p\right)$, 7.09 (d, $\left.{ }^{3} J 8.5 \mathrm{~Hz}, 1 \mathrm{H}, \mathrm{H}-3\right), 6.95$ (td, ${ }^{3} J 7.3$ and ${ }^{4} J 1.6 \mathrm{~Hz}$, 1H, H-5), 6.88 (d, $\left.{ }^{3} J 18.0 \mathrm{~Hz}, 1 \mathrm{H}, \mathrm{CH}-\mathrm{B}\right), 6.41$ (d, ${ }^{2} J 18.0$ $\mathrm{Hz}, 1 \mathrm{H}, \mathrm{C} \underline{\mathrm{H}}-\mathrm{CHB}$ ), 3.89 (d, $\left.{ }^{3} \mathrm{~J} 10.7 \mathrm{~Hz}, 1 \mathrm{H}, \mathrm{H}-9 \mathrm{a}\right), 3.68$ (dd, ${ }^{3} J 10.3$ and $\left.{ }^{3} J 3.3 \mathrm{~Hz}, 1 \mathrm{H}, \mathrm{H}-8 \mathrm{a}\right), 3.60\left(\mathrm{dd},{ }^{3} J 10.7\right.$ and $^{2} J 3.3$ $\mathrm{Hz}, 1 \mathrm{H}, \mathrm{H}-8 \mathrm{~b}$ ), 3.42 (dd, ${ }^{3} J 10.3$ and ${ }^{3} \mathrm{~J} 3.3 \mathrm{~Hz}, 1 \mathrm{H}, \mathrm{H}-9 \mathrm{~b}$ ). ${ }^{13} \mathrm{C}$ NMR (75 MHz, $\left.\mathrm{CDCl}_{3}\right) \delta 164.6(\mathrm{C}-7), 161.1(\mathrm{C}-2)$, 139.7 (C-i), 138.9 (ㄷH-CHB), 137.9 (C-4), 131.5 (C-6), $128.6(\mathrm{C}-m), 127.3(\mathrm{C}-p), 126.7$ (C-o), $119.4(\mathrm{C}-3), 119.2$ (C-5), 116.2 (C-1), 60.8 (C-8), 60.1 (C-9). ${ }^{11}$ B NMR (96 $\left.\mathrm{MHz}, \mathrm{CDCl}_{3}\right) \delta 6.6\left(\mathrm{br}, \mathrm{h}_{1 / 2}=356 \mathrm{~Hz}\right) . \mathrm{m} / \mathrm{z}(\%), 451[\mathrm{M}-$ $\mathrm{CHCHPh}^{+}, 12$ ), 380 (20), 276 (10), 174 (100), 130 (18).

$\left[\mathrm{MeLBCH}=\mathrm{CHPh}_{2}(3 \mathrm{~b}) .(0.26 \mathrm{~g}, 64 \%)\right.$; mp 185$187^{\circ} \mathrm{C}$; Found: C, 73.14; H, 6.58; N, 4.87\%. Calc. for $\mathrm{C}_{36} \mathrm{H}_{36} \mathrm{~B}_{2} \mathrm{~N}_{2} \mathrm{O}_{4}: \mathrm{C}, 74.25 ; \mathrm{H}, 6.18 ; \mathrm{N}, 4.81 \%$; IR $v_{\max } / \mathrm{cm}^{-1}$ : 2944, 2918, $1612(\mathrm{C}=\mathrm{N}), 1554,1446,1330,1276,1146$, 1122, 990 (KBr); ${ }^{1} \mathrm{H}$ NMR $\left(300 \mathrm{MHz}, \mathrm{CDCl}_{3}\right) \delta 7.73\left(\mathrm{~d},{ }^{3} \mathrm{~J}\right.$ $7.3 \mathrm{~Hz}, 1 \mathrm{H}, \mathrm{H}-6), 7.48$ (t, $\left.{ }^{3} J 7.3 \mathrm{~Hz}, 1 \mathrm{H}, \mathrm{H}-4\right), 7.39$ (d, ${ }^{3} J 7.5$ $\mathrm{Hz}, 2 \mathrm{H}, \mathrm{H}-\mathrm{o}), 7.28$ (t, $\left.{ }^{3} \mathrm{~J} 7.5 \mathrm{~Hz}, 2 \mathrm{H}, \mathrm{H}-m\right), 7.18\left(\mathrm{t},{ }^{3} \mathrm{~J} 7.5 \mathrm{~Hz}\right.$, $1 \mathrm{H}, \mathrm{H}-p), 7.08\left(\mathrm{~d},{ }^{3} J 7.3 \mathrm{~Hz}, 1 \mathrm{H}, \mathrm{H}-3\right), 6.92\left(\mathrm{t},{ }^{3} J 7.3 \mathrm{~Hz}, 1 \mathrm{H}\right.$, $\mathrm{H}-5), 6.86$ ( d, $\left.{ }^{3} J 17.8 \mathrm{~Hz}, 1 \mathrm{H}, \mathrm{CH}-\mathrm{B}\right), 6.38\left(\mathrm{~d},{ }^{3} J 17.9 \mathrm{~Hz}\right.$, $1 \mathrm{H}, \mathrm{C} \underline{\mathrm{H}}-\mathrm{CHB}$ ), 4.10 (ddd, ${ }^{3} J 17.9,{ }^{2} J 3.7,{ }^{3} \mathrm{~J} 2.9 \mathrm{~Hz}, 1 \mathrm{H}, \mathrm{H}-$ 9a), 3.61 ( ddd, ${ }^{3} J 17.9,{ }^{2} J 3.7$ and ${ }^{3} J 2.9 \mathrm{~Hz}, 1 \mathrm{H}, \mathrm{H}-8 \mathrm{a}$ ), 3.50 (dd, ${ }^{3} J 12.5$ and ${ }^{2} J 3.7 \mathrm{~Hz}, 1 \mathrm{H}, \mathrm{H}-8 \mathrm{~b}$ ), 3.36 (dd, ${ }^{3} J 12.5$ and $\left.{ }^{2} J 3.7 \mathrm{~Hz}, 1 \mathrm{H}, \mathrm{H}-9 \mathrm{~b}\right) .{ }^{13} \mathrm{C} \mathrm{NMR}\left(75 \mathrm{MHz}, \mathrm{CDCl}_{3}\right) \delta 171.9$ (C-7), 159.8 (C-2), 139.5 (C-i), 137.6 (ㅁH-CHB) 136.1 (C4), 128.4 (C-6), 128.2 (C-m), 126.7 (C- $p), 126.3$ (C-o), 120.0 (C-3), 118.6 (C-5), 117.9 (C-1), 60.9 (C-9), 51.4 (C8), $16.70(\mathrm{Me}) .{ }^{11} \mathrm{~B} \mathrm{NMR}\left(96 \mathrm{MHz}, \mathrm{CDCl}_{3}\right) \delta 2.7\left(\mathrm{br}, \mathrm{h}_{1 / 2}=\right.$ $472 \mathrm{~Hz}$ ). $\mathrm{m} / \mathrm{z}$ (\%), 479 ([M- CHCHPh] $\left.{ }^{+}, 10\right), 395$ (10), 368 (15), 313 (14), 188 (100), 160 (30).

$[\mathrm{PhLBCH}=\mathrm{CHPh}]_{2}(\mathbf{3 c}) .(0.31 \mathrm{~g}, 85 \%) ; \mathrm{mp} 253-255^{\circ} \mathrm{C}$; Found: C, 77.59; H, 5.99; N, 3.97\%; Calc. for $\mathrm{C}_{46} \mathrm{H}_{40} \mathrm{~B}_{2} \mathrm{~N}_{2} \mathrm{O}_{4}: \mathrm{C}, 78.23 ; \mathrm{H}, 5.66 ; \mathrm{N}, 3.96 \%$; IR $v_{\max } / \mathrm{cm}^{-1}$ : 2990, 2932, 2848, 2836, $1608(\mathrm{C}=\mathrm{N}), 1554,1550,1366$, 1152, 1010, $756(\mathrm{KBr}) ;{ }^{1} \mathrm{H}$ NMR $\left(300 \mathrm{MHz}, \mathrm{CDCl}_{3}\right)$ $\delta 7.66\left(\mathrm{~d},{ }^{3} J 6.5 \mathrm{~Hz}, 2 \mathrm{H}, \mathrm{H}-o_{(\mathrm{Ph}-\mathrm{CHN})}\right), 7.60\left(\mathrm{t},{ }^{3} J 6.5 \mathrm{~Hz}, 1 \mathrm{H}\right.$, $\left.\mathrm{H}-p_{(\mathrm{Ph}-\mathrm{CHN})}\right), 7.54$ (td, ${ }^{3} J 7.8$ and $\left.{ }^{3} J 1.5 \mathrm{~Hz}, 1 \mathrm{H}, \mathrm{H}-4\right), 7.47$ (t, $\left.{ }^{3} J 6.5 \mathrm{~Hz}, 1 \mathrm{H}, \mathrm{H}-m_{\text {(Ph-CHN) }}\right), 7.42\left(\mathrm{dd},{ }^{3} J 7.8\right.$ and ${ }^{4} J 1.5 \mathrm{~Hz}$, $1 \mathrm{H}, \mathrm{H}-6), 7.41\left(\mathrm{~d},{ }^{3} J 7.2 \mathrm{~Hz}, 2 \mathrm{H}, \mathrm{H}-o_{(\mathrm{Ph}-\mathrm{CHCH})}\right), 7.27\left(\mathrm{t},{ }^{3} J 7.2\right.$ $\left.\mathrm{Hz}, 2 \mathrm{H}, \mathrm{H}-m_{\text {(Ph-CHCH) }}\right), 7.17$ (td, ${ }^{3} J 7.2$ and ${ }^{4} J 1.5 \mathrm{~Hz}, 1 \mathrm{H}, \mathrm{H}-$ $\left.p_{\text {(Ph-CHCH) }}\right), 7.06\left(\mathrm{~d},{ }^{3} J 18.0 \mathrm{~Hz}, 1 \mathrm{H}, \mathrm{C} \underline{\mathrm{H}}-\mathrm{B}\right), 6.82\left(\mathrm{dd},{ }^{3} J 7.8\right.$ and $\left.{ }^{4} J 1.5 \mathrm{~Hz}, 1 \mathrm{H}, \mathrm{H}-3\right), 6.75$ (td, ${ }^{3} J 7.2$ and ${ }^{4} J 1.5 \mathrm{~Hz}, 1 \mathrm{H}$, H-5), 6.34 (d, $\left.{ }^{3} J 18.0 \mathrm{~Hz}, 1 \mathrm{H}, \mathrm{C} \underline{\mathrm{H}}-\mathrm{CHB}\right), 4.06$ (dd, ${ }^{3} \mathrm{~J} 11.4$ and ${ }^{2} J 4.4 \mathrm{~Hz}, 1 \mathrm{H}, \mathrm{H}-9 \mathrm{a}$ ), 3.3.89 (ddd, ${ }^{3} J 16.7,{ }^{3} J 4.4$ and
${ }^{2} J 3.3 \mathrm{~Hz}, 1 \mathrm{H}, \mathrm{H}-8 \mathrm{a}$ ), 3.49 (ddd, ${ }^{3} J 16.7,{ }^{3} J 4.4,{ }^{2} J 3.3 \mathrm{~Hz}$, 1H, H-8b), 3.36 (dd, $\left.{ }^{3} \mathrm{~J} 11.4,{ }^{2} \mathrm{~J} 4.4 \mathrm{~Hz}, \mathrm{H}-9 \mathrm{~b}\right) .{ }^{13} \mathrm{C}$ NMR $(75$ $\left.\mathrm{MHz}, \mathrm{CDCl}_{3}\right) \delta 174.3(\mathrm{C}-7), 160.8(\mathrm{C}-2), 139.9\left(\mathrm{C}-i_{(\mathrm{Ph}-\mathrm{CHCH}}\right)$, 138.2 ( $\underline{\mathrm{CH}}-\mathrm{CHB}), 136.7$ (C-4), 132.7 (C- $\left.i_{(\mathrm{Ph}-\mathrm{CHN})}\right), 130.2$ $(\mathrm{C}-6), 129.4\left(\mathrm{C}-p_{(\mathrm{Ph}-\mathrm{CHN})}\right), 128.6\left(\mathrm{C}-o_{(\mathrm{Ph}-\mathrm{CHN})}\right), 128.2$ $\left(\mathrm{C}-m_{\text {(Ph-CHCH) }}\right), 127.5\left(\mathrm{C}-m_{(\mathrm{Ph}-\mathrm{CHN})}\right), 127.2\left(\mathrm{C}-p_{(\mathrm{Ph}-\mathrm{CHCH})}\right), 126.7$ $\left(\mathrm{C}-o_{(\mathrm{Ph}-\mathrm{CHCH}}\right), 120.9(\mathrm{C}-3), 119.0(\mathrm{C}-5), 118.5(\mathrm{C}-1), 64.4$ (C-9), 53.6 (C-8). ${ }^{11} \mathrm{~B}$ NMR (96 MHz, $\mathrm{CDCl}_{3}$ ) $\delta 6.5$ (br, $\left.\mathrm{h}_{1 / 2}=389 \mathrm{~Hz}\right) \cdot \mathrm{m} / \mathrm{z}(\%), 603\left([\mathrm{M}-\mathrm{CHCHPh}]^{+}, 10\right), 380(13)$, 352 (15), 276 (14), 250 (100), 174 (20), 91 (10).

[HLBMe] ${ }_{2}(3 \boldsymbol{d}) .(0.52 \mathrm{~g}, 83 \%) ; \mathrm{mp} 298-301{ }^{\circ} \mathrm{C}$; Found: C, 63.12; H, 6.05; N, 7.09\%. Calc. for $\mathrm{C}_{20} \mathrm{H}_{24} \mathrm{~B}_{2} \mathrm{~N}_{2} \mathrm{O}_{4}: \mathrm{C}$, 63.56, H, 6.36; N, 7.41\%; IR $v_{\max } / \mathrm{cm}^{-1}: 2942,1638(\mathrm{C}=\mathrm{N})$, 1480, 1304, 1142, 1126, 1028, 1008, 764 (KBr); ${ }^{1} \mathrm{H}$ NMR (300 MHz, MeOD-d $) \delta 8.46$ (s, 1H, H-7), $7.35\left(\mathrm{dd},{ }^{3} J 7.2\right.$ and $\left.{ }^{4} J 1.8 \mathrm{~Hz}, 1 \mathrm{H}, \mathrm{H}-6\right), 7.32\left(\mathrm{td},{ }^{3} J 7.2\right.$ and ${ }^{4} J 1.8 \mathrm{~Hz}, 1 \mathrm{H}$, H-4), 6.85 (d, $\left.{ }^{3} J 7.2 \mathrm{~Hz}, 1 \mathrm{H}, \mathrm{H}-3\right), 6.84\left(\mathrm{t},{ }^{3} J 7.2 \mathrm{~Hz}, 1 \mathrm{H}, \mathrm{H}-\right.$ 5), $3.84\left(\mathrm{t},{ }^{3} \mathrm{~J} 4.3 \mathrm{~Hz}, 2 \mathrm{H}, \mathrm{H}-9 \mathrm{a}, 8 \mathrm{a}\right), 3.74\left(\mathrm{t},{ }^{3} \mathrm{~J} 4.3 \mathrm{~Hz}, 2 \mathrm{H}, \mathrm{H}-\right.$ 8b, 9b), 2.27 (s, 3H, Me). ${ }^{13} \mathrm{C}$ NMR (75 MHz, MeOD-d $\left.\mathrm{d}_{4}\right) \delta$ 168.4 (C-7), 161.3 (C-2), 134.2 (C-4), 133.2 (C-6), 119.9 (C-5), 119.2 (C-3), 118.6 (C-1), 62.5 (C-8), 61.6 (C-9). ${ }^{11} \mathrm{~B}$ NMR (96 MHz, MeOD-d $\left.{ }_{4}\right) \delta 8.8\left(\mathrm{br}, \mathrm{h}_{1 / 2}=89 \mathrm{~Hz}\right) . \mathrm{m} / z(\%)$, 363 ([M-Me $\left.]^{+}, 12\right), 333$ (10), 190 (18), 188 (25), 174 (100), 159 (16), 132 (14).

[MeLBMe] 2 (3e). (0.45g, 75\%); mp 184-186 ${ }^{\circ} \mathrm{C}$; Found: C, 64.93; H, 7.13; N, $6.88 \%$. Calc. for $\mathrm{C}_{22} \mathrm{H}_{28} \mathrm{~B}_{2} \mathrm{~N}_{2} \mathrm{O}_{4}$ : C, 65.09; H, 6.90; N, $6.90 \%$; IR $v_{\text {max }} / \mathrm{cm}^{-1}: 2954,2908,1618$ $(\mathrm{C}=\mathrm{N}), 1560,1360,1134,116,1010,754(\mathrm{KBr}) ;{ }^{1} \mathrm{H}$ NMR $\left(300 \mathrm{MHz}, \mathrm{CDCl}_{3}\right) \delta 7.64\left(\mathrm{dd},{ }^{3} J 7.8\right.$ and ${ }^{4} J 1.5 \mathrm{~Hz}, 1 \mathrm{H}, \mathrm{H}-$ 6), $7.45\left(\mathrm{td},{ }^{3} J 7.8\right.$ and $\left.{ }^{4} J 1.5 \mathrm{~Hz}, 1 \mathrm{H}, \mathrm{H}-4\right), 7.03\left(\mathrm{dd},{ }^{3} J 7.8\right.$ and $\left.{ }^{4} J 1.5 \mathrm{~Hz}, 1 \mathrm{H}, \mathrm{H}-3\right), 6.88\left(\mathrm{td},{ }^{3} J 7.8\right.$ and ${ }^{4} J 1.5 \mathrm{~Hz}, 1 \mathrm{H}$, H-5), 4.22 (dd, ${ }^{3} J 7.1$ and ${ }^{2} J 3.0 \mathrm{~Hz}, 1 \mathrm{H}, \mathrm{H}-9 \mathrm{a}$ ), 4.05 (ddd, ${ }^{3} \mathrm{~J}$ 9.4, ${ }^{3} J 7.1$ and $\left.{ }^{2} J 3.0 \mathrm{~Hz}, 1 \mathrm{H}, \mathrm{H}-8 \mathrm{a}\right), 3.41$ (dd, ${ }^{3} \mathrm{~J} 7.1,{ }^{2} J 3.0$ $\mathrm{Hz}, 1 \mathrm{H}, \mathrm{H}-8 \mathrm{~b}$ ), 3.31 (dd, ${ }^{3} J 7.1$ and ${ }^{2} J 3.0 \mathrm{~Hz}, 1 \mathrm{H}, \mathrm{H}-9 \mathrm{~b}$ ), 2.69 (s, 3H, C7- $\underline{\mathrm{Me}}$ ), 2.18 (s, 3H, B-Me). ${ }^{13} \mathrm{C}$ NMR (75 $\mathrm{MHz}, \mathrm{CDCl}_{3}$ ) $\delta 171.5$ (C-7), 159.8 (C-2), 136.0 (C-4), 135.8 (C-6), 119.8 (C-3), 118.5 (C-1), 118.1 (C-5), 61.2 (C-9), 51.0 (C-8), 16.4 (C7- $\underline{\mathrm{Me}}) .{ }^{11} \mathrm{~B}$ NMR $\left(96 \mathrm{MHz}, \mathrm{CDCl}_{3}\right) \delta 11$ (br, $\left.\mathrm{h}_{1 / 2}=91 \mathrm{~Hz}\right) . \mathrm{m} / z(\%), 391\left([\mathrm{M}-\mathrm{Me}]^{+}, 30\right), 204(8), 203$ (20), 188 (100), 187 (39), 173 (10), 164 (10), 77 (19).

$\left[\mathrm{PhLBMe}_{2}(3 \mathrm{f}) .(0.13 \mathrm{~g}, 77 \%)\right.$; mp 269-271 ${ }^{\circ} \mathrm{C}$; Found: C, 71.93; H, 6.19; N, 5.10\%. Calc. for $\mathrm{C}_{32} \mathrm{H}_{32} \mathrm{~B}_{2} \mathrm{~N}_{2} \mathrm{O}_{4}: \mathrm{C}$, 72.51; H, 6.04; N, $5.29 \%$; IR $v_{\max } / \mathrm{cm}^{-1}: 2936,1612(\mathrm{C}=\mathrm{N})$, 1594, 1552, 1474, 1364, 1276, 1036 (KBr); ${ }^{1} \mathrm{H}$ NMR (300 $\left.\mathrm{MHz}, \mathrm{CDCl}_{3}\right) \delta 7.76-758(\mathrm{~m}, 2 \mathrm{H}, \mathrm{H}-\mathrm{m}), 7.53\left(\mathrm{dd},{ }^{3} \mathrm{~J} 7.1\right.$ and $\left.{ }^{4} J 1.3 \mathrm{~Hz}, 1 \mathrm{H}, \mathrm{H}-6\right), 7.49\left(\mathrm{td},{ }^{3} J 7.1\right.$ and $\left.{ }^{4} J 1.3 \mathrm{~Hz}, 1 \mathrm{H}, \mathrm{H}-4\right)$, 7.25-7.20 (m, 1H, H-p), 7.10 (dd, ${ }^{3} J 7.1$ and ${ }^{4} J 1.3 \mathrm{~Hz}, 1 \mathrm{H}$, H-3), 7.03 (dd, ${ }^{3} J 7.9$ and ${ }^{4} J 1.7 \mathrm{~Hz}, 2 \mathrm{H}, \mathrm{H}-\mathrm{o}$ ), 6.80 (td, ${ }^{3} J$ 7.1 and ${ }^{4} J 1.3 \mathrm{~Hz}, 1 \mathrm{H}, \mathrm{H}-5$ ), 4.15 (dd, ${ }^{3} J 6.6$ and ${ }^{3} \mathrm{~J} 4.2 \mathrm{~Hz}$, 1H, H-9a), 4.02 (dd, ${ }^{3} J 6.6$ and ${ }^{3} J 4.2 \mathrm{~Hz}, 1 \mathrm{H}, \mathrm{H}-8 \mathrm{a}$ ), 3.90 (dd, ${ }^{3} J 11.6$ and ${ }^{3} J 4.2 \mathrm{~Hz}, 1 \mathrm{H}, \mathrm{H}-8 \mathrm{~b}$ ), 3.51 (dd, ${ }^{2} J 11.6$ and 
$\left.{ }^{2} J 4.2 \mathrm{~Hz}, 1 \mathrm{H}, \mathrm{H}-9 \mathrm{~b}\right), 2.19$ (s, 3H, B-Me). ${ }^{13} \mathrm{C}$ NMR $(75$ $\left.\mathrm{MHz}, \mathrm{CDCl}_{3}\right) \delta 170.7(\mathrm{C}-7), 161.6(\mathrm{C}-2), 132.0(\mathrm{C}-4), 131.3$ (C-6), 130.6 (C-i), 129.3 (C-m), 129.0 (C-p) 128.4 (C-o), 120.5 (C-5), 118.7 (C-1), 118.4 (C-3), 63.8 (C-9), 52.2 (C8). ${ }^{11} \mathrm{~B} \mathrm{NMR}\left(96 \mathrm{MHz}, \mathrm{CDCl}_{3}\right) \delta 11.5\left(\mathrm{br}, \mathrm{h}_{1 / 2}=120 \mathrm{~Hz}\right)$. $\mathrm{m} / \mathrm{z}$ (\%), 515 ([M-Ph] $\left.{ }^{+}, 20\right), 267$ (18), 266 (12), 265 (40), 250 (100), 234 (27), 222 (19), 146 (13).

$\left[\mathrm{HLB}\left(3-\mathrm{C}_{4} \mathrm{H}_{3} \mathrm{~S}\right)\right]_{2}(3 \mathrm{~g}) .(1.06 \mathrm{~g}, 68 \%) ; \mathrm{mp} 280-281^{\circ} \mathrm{C}$; Found: $\mathrm{C}, 60.87 ; \mathrm{H}, 4.37, \mathrm{~N}, 4.89 \%$; Calc. for $\mathrm{C}_{26} \mathrm{H}_{24} \mathrm{~B}_{2} \mathrm{~N}_{2} \mathrm{O}_{4} \mathrm{~S}_{2}: \mathrm{C}, 60.70 ; \mathrm{H}, 4.67, \mathrm{~N}, 5.44 \%$; IR $v_{\max } / \mathrm{cm}^{-1}$ : 2966, 2930, 2850, $1640(\mathrm{C}=\mathrm{N}), 1558,1312,1210,1156$, $1148,1140,1122,1026,988,772(\mathrm{KBr}) ;{ }^{1} \mathrm{H}$ NMR $(300$ $\mathrm{MHz}$ DMSO-d $\left.{ }_{6}\right) \delta 8.29(\mathrm{~s}, 1 \mathrm{H}, \mathrm{H}-7), 7.73\left(\mathrm{dd},{ }^{3} J 7.7\right.$ and ${ }^{4} \mathrm{~J}$ $1.6 \mathrm{~Hz}, 1 \mathrm{H}, \mathrm{H}-6$ ), 7.56 (ddd, ${ }^{3} J 7.8,{ }^{3} \mathrm{~J} 7.2$ and ${ }^{4} \mathrm{~J} 1.7 \mathrm{~Hz}, 1 \mathrm{H}$, $\mathrm{H}-4), 7.31$ (dd, ${ }^{3} J 4.6$ and $\left.{ }^{4} J 2.8 \mathrm{~Hz}, 1 \mathrm{H}, \mathrm{H}-13\right), 7.27$ (dd, ${ }^{4} J$ 2.8 and $\left.{ }^{4} J 1.1 \mathrm{~Hz}, 1 \mathrm{H}, \mathrm{H}-11\right), 7.01$ (dd, ${ }^{3} J 4.6$ and ${ }^{4} J 1.1 \mathrm{~Hz}$, 1H, H-12), 6.96 (td, ${ }^{3} J 7.0$ and $\left.{ }^{4} J 1.5 \mathrm{~Hz}, 1 \mathrm{H}, \mathrm{H}-5\right), 6.90$ (d, ${ }^{3} \mathrm{~J} 7.6 \mathrm{~Hz}, 1 \mathrm{H}, \mathrm{H}-3$ ), 3.02-3.52 (m, 4H, H-8,9). ${ }^{13} \mathrm{C} \mathrm{NMR}(75$ MHz, DMSO-d 6 ) $\delta 166.5$ (C-7), 160.6 (C-2), 138.1 (C-4), 132.7 (C-12), 132.3 (C-6), 128.1 (C-11), 125.1 (C-13) 119.5 (C-3), 118.6 (C-5), 117.0 (C-1), 60.1 (C-8), 59.9 (C-9) ppm. ${ }^{11} \mathrm{~B}$ NMR $\left(96 \mathrm{MHz}\right.$, DMSO-d 6 ) $\delta 7.0\left(\mathrm{br}, \mathrm{h}_{1 / 2}=904 \mathrm{~Hz}\right)$. $\mathrm{m} / \mathrm{z}(\%), 431\left(\left[\mathrm{M}-\mathrm{C}_{4} \mathrm{H}_{3} \mathrm{~S}\right]^{+}, 22\right), 258$ (17), 226 (18), 174 (100), 148 (9), 77 (20).

$\left[\mathrm{MeLB}\left(3-\mathrm{C}_{4} \mathrm{H}_{3} \mathrm{~S}\right)\right]_{2}(3 \boldsymbol{h}) .(0.70 \mathrm{~g}, 93 \%) ; \mathrm{mp} 198-200{ }^{\circ} \mathrm{C}$; Found: $\mathrm{C}, 61.37 ; \mathrm{H}, 5.41 ; \mathrm{N}, 4.98 \%$. Calc. for $\mathrm{C}_{28} \mathrm{H}_{28} \mathrm{~B}_{2} \mathrm{~N}_{2} \mathrm{O}_{4} \mathrm{~S}_{2}: \mathrm{C}, 61.99 ; \mathrm{H}, 5.16 ; \mathrm{N}, 5.16 \%$; IR $v_{\max } / \mathrm{cm}^{-1}$ : 2918, 2850, 2362, 2344, $1636(\mathrm{C}=\mathrm{N}), 1516,1472,1436$, 1414, 1380, 1328, 1174, 674 (KBr); ${ }^{1} \mathrm{H}$ NMR $(300 \mathrm{MHz}$, $\left.\mathrm{CDCl}_{3}\right) \delta 7.80\left(\mathrm{dd},{ }^{3} J 8.2\right.$ and $\left.{ }^{4} J 1.5 \mathrm{~Hz}, 1 \mathrm{H}, \mathrm{H}-6\right), 7.51$ (ddd, ${ }^{3} J 8.2,{ }^{3} J 7.0$ and $\left.{ }^{4} J 1.5 \mathrm{~Hz}, 1 \mathrm{H}, \mathrm{H}-4\right), 7.28\left(\mathrm{dd},{ }^{4} J 2.7\right.$ and ${ }^{4} J$ $1.1 \mathrm{~Hz}, 1 \mathrm{H}, \mathrm{H}-11), 7.23$ (dd, $\left.{ }^{3} \mathrm{~J} 4.6^{4} \mathrm{~J} 2.7 \mathrm{~Hz}, 1 \mathrm{H}, \mathrm{H}-13\right), 7.09$ (dd, ${ }^{3} J 7.0$ and $\left.{ }^{4} J 1.5 \mathrm{~Hz}, 1 \mathrm{H}, \mathrm{H}-3\right), 7.08\left(\mathrm{dd},{ }^{3} J 4.6\right.$ and ${ }^{3} J 1.1$ $\mathrm{Hz}, 1 \mathrm{H}, \mathrm{H}-12$ ), 6.98 (ddd, ${ }^{3} J 8.2,{ }^{3} J 7.0$ and ${ }^{4} J 1.5 \mathrm{~Hz}, 1 \mathrm{H}, \mathrm{H}-$ 5), 4.01 (td, ${ }^{3} J 12.3$ and $\left.{ }^{3} J 5.1 \mathrm{~Hz}, 1 \mathrm{H}, \mathrm{H}-9 \mathrm{a}\right), 3.66$ (dt, ${ }^{3} J 10.2$ and ${ }^{3} J 3.9 \mathrm{~Hz}, 1 \mathrm{H}, \mathrm{H}-8 \mathrm{a}$ ), 3.34 (dd, ${ }^{3} J 10.2$ and ${ }^{3} J 5.1 \mathrm{~Hz}, \mathrm{H}-$ 8b), 3.07 (dd, ${ }^{3} J 12.3$ and $\left.{ }^{3} J 3.9 \mathrm{~Hz}, 1 \mathrm{H}, \mathrm{H}-9 \mathrm{~b}\right), 2.90$ (s, 3H, Me) ppm. ${ }^{13} \mathrm{C} \mathrm{NMR}\left(75 \mathrm{MHz}, \mathrm{CDCl}_{3}\right) \delta 172.5$ (C-7), 160.2 (C-2), 136.7 (C-4), 131.7 (C-12), 128.9 (C-6), 128.0 (C-11), 124.8 (C-13) 120.4 (C-3), 119.1 (C-5), 118.1 (C-1), 61.4 (C-9), $51.6(\mathrm{C}-8), 17.2(\mathrm{Me}) .{ }^{11} \mathrm{~B}$ NMR $\left(96 \mathrm{MHz}, \mathrm{CDCl}_{3}\right) \delta$ $6.0\left(\mathrm{br}, \mathrm{h}_{1 / 2}=210 \mathrm{~Hz}\right) . \mathrm{m} / z(\%), 459\left(\left[\mathrm{M}-\mathrm{C}_{4} \mathrm{H}_{3} \mathrm{~S}\right]^{+}, 40\right), 296$ (15), 240 (35), 188 (100), 162 (28), 146 (24), 77 (45).

$\left[\mathrm{PhLB}\left(3-\mathrm{C}_{4} \mathrm{H}_{3} \mathrm{~S}\right)\right]_{2}(3 \mathrm{i}) .(0.26 \mathrm{~g}, 75 \%) ; \mathrm{mp} 243-245{ }^{\circ} \mathrm{C}$; Found: $\mathrm{C}, 68.25 ; \mathrm{H}, 5.05 ; \mathrm{N}, 4.16 \%$. Calc. for $\mathrm{C}_{38} \mathrm{H}_{32} \mathrm{~B}_{2} \mathrm{~N}_{2} \mathrm{O}_{4} \mathrm{~S}_{2}: \mathrm{C}, 68.51 ; \mathrm{H}, 4.80 ; \mathrm{N}, 4.20 \%$; IR $v_{\text {max }} / \mathrm{cm}^{-1}$ : 2936, 2916, $1606(\mathrm{C}=\mathrm{N}), 1550,1472,1208,1152,1024$, $760(\mathrm{KBr}) ;{ }^{1} \mathrm{H}$ NMR $\left(300 \mathrm{MHz}, \mathrm{CDCl}_{3}\right) \delta 7.64\left(\mathrm{t},{ }^{3} \mathrm{~J}=7.2\right.$ $\mathrm{Hz}, 2 \mathrm{H}, \mathrm{H}-o), 7.58\left(\mathrm{t},{ }^{3} J 7.2 \mathrm{~Hz}, 2 \mathrm{H}, \mathrm{H}-m\right), 7.52\left(\mathrm{~d},{ }^{3} J 7.6 \mathrm{~Hz}\right.$, $1 \mathrm{H}, \mathrm{H}-6), 7.28\left(\mathrm{t},{ }^{3} J 7.2 \mathrm{~Hz}, 1 \mathrm{H}, \mathrm{H}-p\right), 7.24\left(\mathrm{t},{ }^{3} J 7.6 \mathrm{~Hz}, 1 \mathrm{H}\right.$, H-4), $7.20\left(\mathrm{~d},{ }^{3} J 10.2 \mathrm{~Hz}, 1 \mathrm{H}, \mathrm{H}-13\right), 7.19\left(\mathrm{~d},{ }^{4} \mathrm{~J} 2.6 \mathrm{~Hz}, 1 \mathrm{H}\right.$,
H-11), 7.04 (dd, ${ }^{3} J 10.2$ and $\left.{ }^{4} J 2.6 \mathrm{~Hz}, 1 \mathrm{H}, \mathrm{H}-12\right), 7.02\left(\mathrm{~d},{ }^{3} J\right.$ $7.6 \mathrm{~Hz}, 1 \mathrm{H}, \mathrm{H}-3), 6.83\left(\mathrm{t},{ }^{3} J 7.6 \mathrm{~Hz}, 1 \mathrm{H}, \mathrm{H}-5\right), 4.28\left(\mathrm{dd},{ }^{3} \mathrm{~J}\right.$ 14.3 and $\left.{ }^{3} J 4.6 \mathrm{~Hz}, 1 \mathrm{H}, \mathrm{H}-9 \mathrm{a}\right), 4.16$ (dd, ${ }^{3} J 11.7$ and ${ }^{3} J 3.2$ $\mathrm{Hz}, 1 \mathrm{H}, \mathrm{H}-8 \mathrm{a}), 4.00$ (dd, ${ }^{3} \mathrm{~J} 14.3$ and ${ }^{3} \mathrm{~J} 4.6 \mathrm{~Hz}, 1 \mathrm{H}, \mathrm{H}-8 \mathrm{~b}$ ), 3.65 (dd, ${ }^{3} J 11.7$ and $\left.{ }^{3} J 3.2 \mathrm{~Hz}, 1 \mathrm{H}, \mathrm{H}-9 \mathrm{~b}\right) .{ }^{13} \mathrm{C}$ NMR $(75$ $\left.\mathrm{MHz}, \mathrm{CDCl}_{3}\right) \delta 181.2(\mathrm{C}-7), 161.3(\mathrm{C}-2), 136.9(\mathrm{C}-4), 132.1$ (C-6), 131.9 (C-i), 131.6 (C-m), 130.8 (C-17), 130.5 (C12), 129.0 (C-o), 128.8 (C-11), 124.2 (C-13) 120.3 (C-5), 119.6 (C-1), 118.8 (C-3), 63.6 (C-9), 52.5 (C-8). ${ }^{11} \mathrm{~B}$ NMR $\left(96 \mathrm{MHz}, \mathrm{CDCl}_{3}\right) \delta 8.9\left(\mathrm{br}, \mathrm{h}_{1 / 2}=142 \mathrm{~Hz}\right) \cdot \mathrm{m} / z(\%), 583([\mathrm{M}-$ $\left.\left.\mathrm{C}_{4} \mathrm{H}_{3} \mathrm{~S}\right]^{+}, 11\right), 523$ (10), 421 (16), 334 (26), 250 (100), 174 (19), 91 (36).

\section{Acknowledgements}

The authors thank the Consejo Nacional de Ciencia y Tecnología (CONACyT, México) for financial support and the Consejo Superior de la Investigación Científica in Spain for the award of a license for the use of the Cambridge Crystallographic Data Base.

\section{References}

1. Caligaris, M.; Randaccio, L. In Comprehensive Coordination Chemistry 2, Wilkinson, G.; Gillard, R.D.; McCleverty, J., eds., Pergamon: Elmsford, New York, 1987; Clague, M.J.; Keder, N.L.; Butler, A.; Inorg. Chem. 1993, 32, 5343; Dutta, S.; Chakratvorty, A.; Polyhedron 1994, 13, 1811; Wong, C.Y.; McDonald, R.; Inorg. Chem. 1996, 35, 325; Tshuva, E.Y.; Versano, M.; Goldberg, I.; Kol, M.; Weitman, H.; Goldschmidt, Z.; Inorg. Chem. Commun. 1999, 2, 371; Janas, Z.; Jerzykiewicz, L.B.; Przybylak, K.; Sobota, P.; Szczegot, K.; Eur. J. Inorg. Chem. 2004, 1639.

2. Wei, P.; Atwood, D.A.; Inorg. Chem. 1998, 37, 4934; Tshuva, E.Y.; Versano, M.; Goldberg, I.; Kol, M.; Weitman, H.; Goldschmidt, Z.; Inorg. Chem. Comm. 1999, 2, 371; Li, Y.; Liu, Y.; Bu, W.; Guo, J.; Wang, Y.; Chem. Commun. 2000 1551; Iwanek, W.; Urbaniak, M.; Gawdzik, B.; Schurig, V.; Tetrahedron: Asymmetry 2003, 14, 2787; Farfán, N.; Mancilla, T.; Santillan, R.; Gutierréz, A.; Zamudio-Rivera, L.S.; Beltrán, H.I.; J. Organomet. Chem. 2004, 689, 3481; Yin, H.D.; Wang, Q.B.; Xue, S.C.; J. Organomet. Chem. 2004, 689, 2480.

3. Höpfl, H.; Sánchez, M.; Farfán, N.; Barba, V.; Can. J. Chem. 1998, 76, 1352; Barba, V.; Cuautle, D.; Santillan, R.; Farfán, N.; Can. J. Chem. 2001, 79, 1229.

4. Höpfl, H.; Farfán, N.; J. Organomet. Chem. 1997, 547, 71; Farfán, N.; Höpfl, H.; Barba, V.; Ochoa, M.E.; Santillan, R.; Gómez, E.; Gutiérrez, A.; J. Organomet. Chem. 1999, 581, 70.

5. Höpfl, H.; Sánchez, M.; Barba, V.; Farfán, N.; Rojas, S.; Santillan, R.; Inorg. Chem. 1998, 37, 1679. 
6. Barba, V.; Luna, R.; Castillo, D.; Santillan, R.; Farfán, N.; J. Organomet. Chem. 2000, 604, 273.

7. Barba, V.; Xochipa, R.; Santillan, R.; Farfán, N.; Eur. J. Inorg. Chem. 2004, 118.

8. Barba, V.; Vargas, G.; Gómez, E.; Farfán, N.; Inorg. Chim. Acta 2000, 311, 133.

9. Grant, D.M.; Cheney, B.V; J. Am. Chem. Soc. 1967, 89, 5315; Wilson, K. In Topics in Stereochemistry 8; Eliel, E.L.; Allinger, N.L., eds., John Wiley and Sons: New York, 1974, p.1-158.

10. Crystal data for 3g: Triclinic, $a=6.4394(10), b=11.8989(10)$, $c=20.1686(10) \AA, a=83.926(10), b=80.839(10), g=$ 74.294(10) $)^{\circ}$, space group $P-1, V=1465.5(3) \AA^{3}, T=293 \mathrm{~K}$, $Z=2, \lambda(\mathrm{Mo}-\mathrm{K} \alpha)=0.71073 \mathrm{~mm}^{-1}, 6166$ reflections measured, 3391 unique, $\left(\mathrm{R}_{\mathrm{int}}=0.03\right), R_{1}[I>2 s I]=0.0588, w R_{2}$ (all data) $=0.2119$.
11. The sum of the van der Waals radii for the hydrogen and oxygen atoms is 2.70 A. Bondi, A.; J. Phys. Chem. 1964, 68, 441.

12. Sheldrick, G.M.; SHELXS-86, Program for Crystal Structure Solution, University of Goettingen: Germany, 1986.

13. Sheldrick, G.M.; SHELXL-97, Program for Crystal Structure Refinement, University of Goettingen: Germany, 1997.

14. Alyea, E.C.; Malek, A.; Can. J. Chem. 1975, 53, 939.

Received: November 4, 2004 Published on the web: April 13, 2005 